\title{
COMPATIBILIDADE ENTRE O MARMELEIRO PORTA-ENXERTO cV. EMC E CULTIVARES DE PEREIRA
}

\section{GRAFTING COMPATIBILITY BETWEEN THE QUINCE ROOTSTOCK CV. EMC AND PEARS CULTIVARS'}

\author{
Clevison Luiz GIACOBBO2 \\ José Carlos FACHINELLO ${ }^{3}$ \\ Luciano PICOLOTTO 4
}

\begin{abstract}
RESUMO
Objetivou-se com este trabalho, verificar o grau de compatibilidade entre o porta-enxerto de marmeleiro cv. EMC e as cultivares de pereira Cascatense, Carrick e Seleta, com três anos de idade. O pomar foi conduzido no sistema de média/alta densidade (2000 plantas ha-1), em forma de líder central e irrigado por gotejamento $\left(2 \mathrm{~L} \mathrm{~h}^{-1}\right.$, por planta). Analisou-se a compatibilidade de enxertia, verificada através do diâmetro do tronco e avaliação visual; a altura de copa e o volume de copa. A área da secção do tronco foi semelhante para todos os tratamentos, exceto quando comparada com o ponto de enxertia, em que o diâmetro deste foi superior ao diâmetro do tronco tanto da cv. copa quanto do porta-enxerto, em todos os tratamentos. Na observação visual da compatibilidade de enxertia, verificou-se que somente na cultivar Cascatense, o porta-enxerto EMC cresceu menos em relação à cultivar copa, ocorrendo hipertrofia acentuada no ponto de enxertia, enquanto que as cultivares Carrick e Seleta, apresentaram visualmente diferenças que podem ser consideradas normais, com leve hipertrofia. Quanto a altura e volume de copa, das plantas, as da cv. Cascatense tiveram

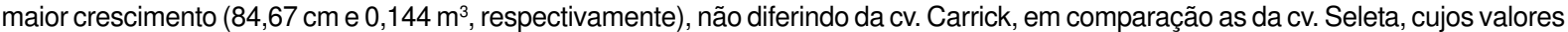

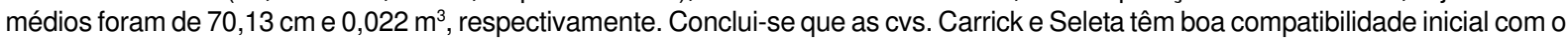
porta-enxerto EMC.
\end{abstract}

Palavras-chave: Cydonia oblonga; Pyrus spp.; incompatibilidade de enxertia.

\begin{abstract}
The aim of this research was to verify the grafting compatibility between pear cultivars Cascatense, Carrick and Seleta and the quince rootstock 'EMC', with three-years old plants. The orchard was growing in field conditions in an high/density system (2000 trees ha-1) and pruned in form of central leader and irrigated with a drip irrigation system ( $2 \mathrm{~L} \mathrm{~h}^{-1}$ emitters per tree). The analyzed parameters were: 1) Compatibility of grafting, verified through the diameter of the trunk and visual evaluation;2) Height of plant and 3 ) Canopy Volume. The diameter of the trunk was similar for all the treatments, except when compared with the grafting point, which had presented a higher diameter in all rootstock/cultivar combinations. By the visual observation of the grafting point, it was verified that only in the interation Cascatense/EMC the rootstock presented a reduced development in relation to cultivar canopy, besides presenting an extreme developed hipertrofia the grafting point. The cultivars Carrick and Seleta had presented smaller differences, which can be considered normal even if a light hipertrofia was observed. The cv. Cascatense presented greater development with $84.67 \mathrm{~cm}$ and $0.144 \mathrm{~m}^{3}$, for height and canopy volume, respectively. On the other hand, the cv. Select showed the lowest development $\left(70.13 \mathrm{~cm} \mathrm{and} 0.022 \mathrm{~m}^{3}\right.$, respectively). The cultivars Carrick and Seleta presented a good grafting compatibility with the EMC quince roostock.

Key-words: Cydonia oblonga; Pyrus spp.; grafting incompatibility.
\end{abstract}

\footnotetext{
${ }^{1}$ Trabalho realizado junto ao PPGA/FAEM/UFPel, com apoio financeiro de FAPERGS/CAPES;

${ }^{2}$ Dr. Bolsista PDJ - CNPq. Depto de Fitotecnia. FAEM/UFPel. Caixa Postal 354. CEP 96010-900. Pelotas, RS. Sala 613. E-mail: giacobboc@yahoo.com.br. Autor para correspondência

${ }^{3}$ Dr. Prof. Titular Departamento de fitotecnia, FAEM/UFPel, jfachi@ufpel.tche.br;

${ }^{4}$ Doutorando do PPGA/CNPq, Fruticultura de Clima Temperado, FAEM/UFPel.
} 


\section{INTRODUÇÃO}

A utilização do marmeleiro como porta-enxerto para a cultura da pereira apresenta inúmeras vantagens, todavia a sua combinação apresenta alguns problemas, como incompatibilidade de enxertia com algumas cultivares podendo ocasionar a quebra de plantas no pomar (LEMOINE et al., 1997).

A incompatibilidade de enxertia é definida como, a incapacidade de formar a união entre o portaenxerto e a copa, incapacidade de uma planta enxertada crescer normalmente, a ocorrência de morte prematura da planta enxertada ou ainda a intolerância fisiológica a nível celular (MOORE, 1986; HARTMANN et al., 1990; SALAYA, 1999). Existem três tipos de incompatibilidade entre porta-enxerto e cultivar: incompatibilidade total, com descontinuidade dos tecidos em correspondência ao ponto de enxertia e sem descontinuidade dos mesmos (FONTANAZZA e BALDONI, 1992). A descontinuidade dos tecidos é freqüentemente mais observada em espécies lenhosas frutíferas, sendo caracterizada pelo maior desenvolvimento da copa em relação ao porta-enxerto que, ao longo dos anos se torna frágil para sustentar a parte aérea, podendo ocorrer a quebra da planta.

A incompatibilidade pode ser relacionada à presença de algum tipo de vírus ou micoplasma existente em uma das duas partes que não é tolerada pela outra (SALAYA, 1999). O autor citou que plantas de pereira cv. Bartlett livres de vírus e enxertadas em marmeleiro apresentaram uma sensível redução nos sintomas de incompatibilidade.

A incompatibilidade de enxertia, também pode estar relacionada com diferenças no nível proteico entre o porta-enxerto e a cultivar copa. MUSACCHI (1996), em estudo sobre incompatibilidade de enxertia de pereira sobre marmeleiro, encontrou diferentes proteínas entre as duas espécies e a presença de diferentes modelos protéicos nos calos unidos por cinco dias na mesma placa de petri em comparação àqueles tecidos das duas espécies que não foram colocados em contato.

FACHINELLO et al. (1999), mediante análise do padrão isoenzimático, observaram que além das alterações a nível fisiológico, ocorre interação celular em nível molecular que permite a manifestação de novas formas isoenzimáticas como resultado da modificação bioquímica da união copa/porta-enxerto.

Em estudos sobre a incompatibilidade de enxertia de algumas cultivares de pereira sobre marmeleiro, CARRERA (1994) verificou que a cultivar Passé Crassane apresentou ótima compatibilidade de enxertia tendo $98 \%$ das plantas enxertadas classificadas como união dos tipos $\mathrm{A}$ e $\mathrm{B}$, segundo a classificação de Herrero. O mesmo concluiu que, em algumas cultivares, se pode notar poucas diferenças no comportamento do enxerto da mesma cultivar quando enxertada sobre diferentes porta-enxertos e verificou que a cv. Spadona Estiva apresentou 11 uniões do Tipo $C$ e 4 do tipo $D$ sobre o marmeleiro EMA, enquanto que com a utilização do EMC apresentou 4 uniões do tipo $C$ e 11 do tipo $D$.
O marmeleiro, porta-enxerto cv. EMC apresenta como principais características: boa capacidade de enraizamento por estaquia; alta contensão de vigor; alta precocidade de início de produção, o que o torna interessante para utilização em pomares de alta densidade de plantio. Porém é incompatível com diversas cultivares de pereira com expressiva importância comercial (BELLINI, 1993).

O objetivo com este trabalho foi determinar o grau de compatibilidade entre o marmeleiro portaenxerto de pereira, cv. EMC e as pereiras cvs. Cascatense, Carrick e Seleta.

\section{MATERIAL E MÉTODOS}

O trabalho foi conduzido no Centro Agropecuário da Palma, da Faculdade de Agronomia Eliseu Maciel (FAEM) - Universidade Federal de Pelotas - UFPel, localizado no Município de Capão do Leão.

Para o trabalho, utilizaram-se três cultivares de pereiras com três anos, sendo Cascatense, Carrick e Seleta, enxertadas sobre o marmeleiro porta-enxerto, cv. EMC. O método de enxertia utilizado foi de dupla fenda inglesa (VALLI, 2002). A cv. Cascatense apresenta vigor médio a semi-vigoroso; a cv. Carrick medianamente vigoroso; e a cv. Seleta semi-vigoroso (SIMONETTO e GRELLMANN, 1999; CAMPODALL'ORTO et al., 1996).

O delineamento estatístico foi em blocos inteiramente casualizados, em esquema fatorial $3 \times 3$, totalizando 9 tratamentos com três repetições de cinco plantas por repetição e três cultivares de pereira.

O pomar foi conduzido no sistema de média/ alta densidade com espaçamentos de 5,0 x 1,0 m (2000 plantas ha ${ }^{-1}$ ) e as plantas conduzidas em forma de líder central e a irrigação realizada por gotejamento. O solo é classificado como moderadamente profundo, com textura média no horizonte $A$ e argilosa no $B$, classificado como Podzólico Vermelho-Amarelo.

As variáveis analisadas foram: 1) Compatibilidade de enxertia, verificada através do: a) diâmetro do tronco - determinado através da mensuração do terceiro ano (mês de abril de cada ano) do diâmetro do tronco no sentido transversal e longitudinal à linha de plantio, com paquímetro digital $(\mathrm{mm})$, a $10 \mathrm{~cm}$ abaixo e $10 \mathrm{~cm}$ acima do ponto da enxertia e da medida do ponto de enxertia; b) avaliação visual - verificado no mês de junho de 2005 através de observação visual de anomalias apresentadas no ponto de enxertia; 2) Altura da planta $(\mathrm{cm})$ : medida a partir do solo até o ápice da planta, no mês de abril de 2005 e 3) Volume de copa: calculado pela multiplicação da largura da planta na fila e na entre fila e altura da planta, medida a partir da emissão das primeiras inserções de ramos e calculado através da fórmula $\mathrm{VC}=$ largura $\mathrm{x}$ espessura $\mathrm{x}$ altura e expresso em $\mathrm{m}^{3}$, realizado no mês de abril.

Os dados foram submetidos à análise da variância pelo teste $F$ e, quando significativo, foi realizada a comparação de médias. As médias dos tratamentos foram comparadas entre si pelo teste de 
Duncan ao nível de $5 \%$ de significância e a análise foi realizada através do programa estatístico WinStat (MACHADO e CONCEIÇÃO, 2002).

\section{RESULTADOS E DISCUSSÃO}

Com relação ao diâmetro do tronco no ponto de enxertia, não foi verificada diferença estatística significativa entre o porta-enxerto e as cultivares copa. Porém, quando comparado o diâmetro no ponto de enxertia com as medidas do tronco a $10 \mathrm{~cm}$ abaixo e acima do mesmo, verificou-se que o crescimento do tronco no ponto de enxertia foi superior ocorrendo um maior intumescimento, diferindo significativamente dos diâmetros do porta-enxerto e o da cultivar copa em todos os tratamentos (Figura 1).

Estes resultados estão de acordo com o descrito por VALLI (2002), o qual definiu esta diferença no ponto de enxertia como incompatibilidade de enxertia, sugerindo como uma das principais causas destas diferenças de diâmetro, a migração da seiva e diferentes coeficientes transpiratórios. O autor cita ainda que podem ser formadas substâncias tóxicas no ponto de enxertia, como exemplo, da prunasina presente no marmeleiro, a qual migra para a pereira, onde sofre hidrólise enzimática, liberando ácido cianídrico o qual pode ocasionar necroses nos vasos lenhosos.

Outra hipótese para a ocorrência desse crescimento no ponto de enxertia, apresentada por RYUGO (1993), seria pelo fato que o câmbio do portaenxerto e da copa, possuindo diferentes taxas de divisão celular, podem diferir na velocidade de formação do xilema e floema. Diferentes taxas de desenvolvimento ocasionam uma hipertrofia ou redução de crescimento no ponto de união que retardam o transporte de nutrientes elaborados na parte superior da copa, prejudicando o desenvolvimento da planta.

Pela observação visual da compatibilidade de enxertia, verificou-se que a combinação Cascatense/EMC manifestou sintoma de incompatibilidade, apresentando hipertrofia acentuada no ponto de enxertia (Figura $2 \mathrm{~A}$ e D). Nas cultivares, Carrick e Seleta, embora apresentando diferenças estatísticas significativas entre as medidas de diâmetro do tronco e do ponto de enxertia, o maior desenvolvimento pode ser considerado normal ou com leve hipertrofia, pela observação visual (Figura 2 B e C, respectivamente). Para VALLI (2002), hipertrofia leve no ponto de enxertia, nem sempre é sintoma de incompatibilidade.

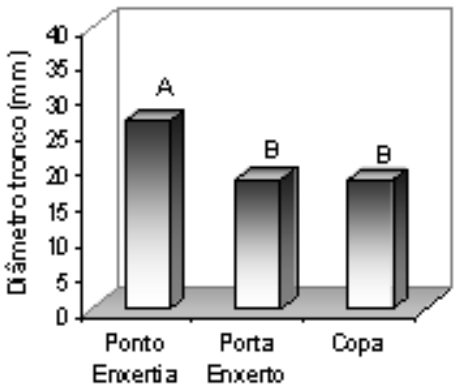

B

FIGURA 1 - Diâmetros médios de pereiras: A) Cascatense; B) Carrick; e C) Seleta, enxertadas sobre marmeleiro cv. EMC, medidos no ponto, $10 \mathrm{~cm}$ abaixo e $10 \mathrm{~cm}$ acima do local da enxertia. FAEM/UFPEL, 2005. * Letras distintas sobre as colunas, diferem entre si pelo teste de Duncan a 5\% de significância. 


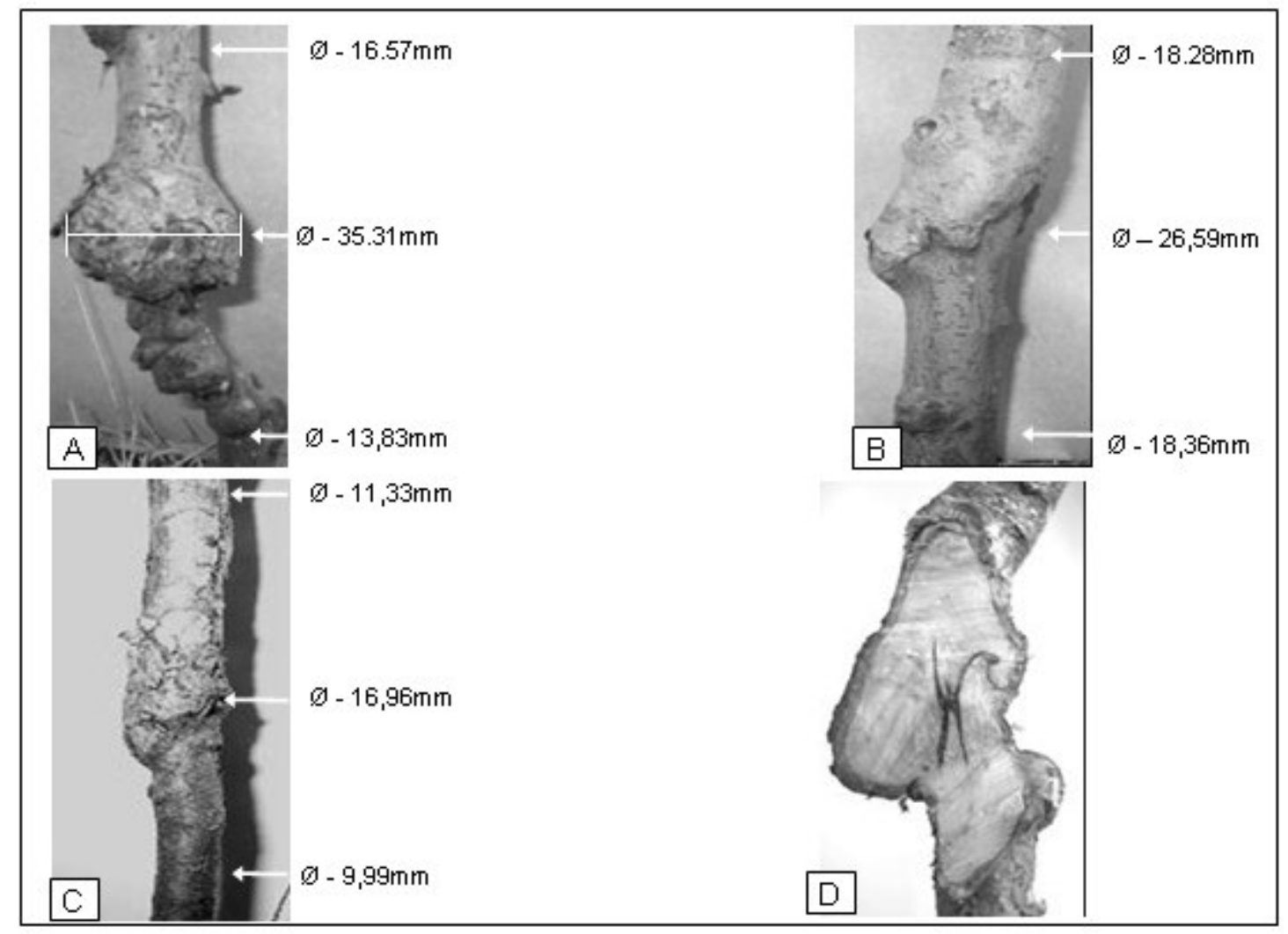

FIGURA 2 - Grau de compatibilidade entre o porta-enxerto de marmeleiro EMC e as cvs. copa de pereira; A) Cascatense; B) Carrick; C) Seleta; e D) secção longitudinal da zona de enxertia da cv. Cascatense sobre EMC, com formação de hipertrofia. FAEM/UFPel, 2005.

Quando utilizado marmeleiro como portaenxerto para pereiras, é freqüente ocorrer incompatibilidade entre as espécies, o que dificulta a utilização dos mesmos. Carrera (1994), em estudo da compatibilidade de enxertia, avaliou 25 cultivares de pereira enxertadas sobre marmeleiro e verificou que somente 6 destas (Abbe Fétel, Beurre Hardy, Doyenné Du Comice, Passa Crassana, Presidente Drouard e Winter Nelis) puderam ser consideradas completamente compatíveis com os porta-enxertos.
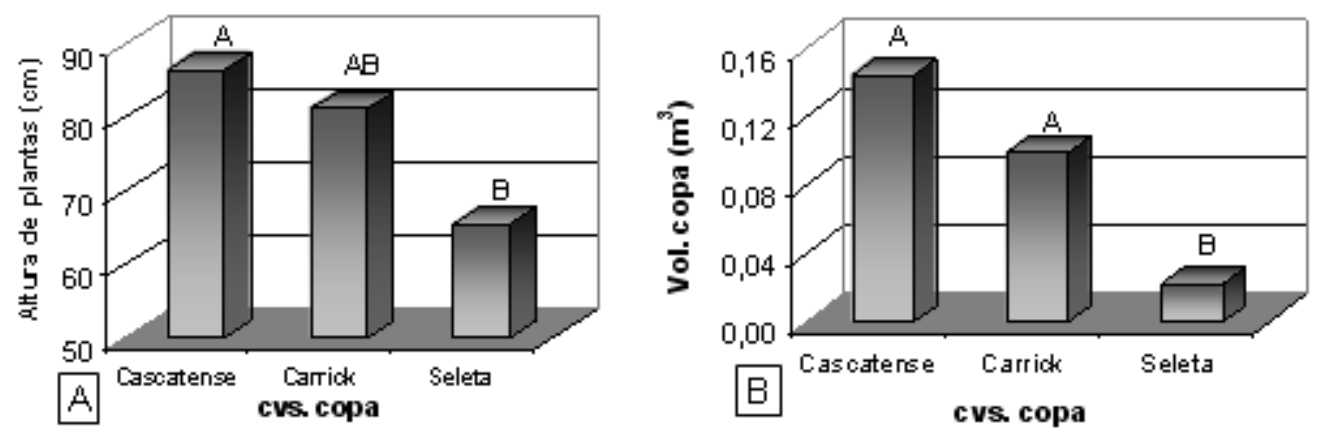

FIGURA 3 - A) altura média e B) volume médio de copa, de pereira cultivares Cascatense, Carrick e Seleta, sobre o porta-enxerto de marmeleiro cv. EMC, ao final de três anos. FAEM/UFPEL, 2005. * Letras diferentes, diferem entre si pelo teste de Duncan a 5\% de significância. 
No que se refere às variáveis altura de planta $e$ volume de copa, embora todas as cultivares tenham apresentado baixo crescimento, a cv. Cascatense teve crescimento superior com $86,40 \mathrm{~cm}$ e $0,144 \mathrm{~m}^{3}$, respectivamente, e a cv. Seleta foi a inferior, com 65,58 $\mathrm{cm} \mathrm{e} 0,022 \mathrm{~m}^{3}$, respectivamente (Figura $3 \mathrm{~A} \mathrm{e} \mathrm{B).} \mathrm{O} \mathrm{maior}$ crescimento da parte aérea na cultivar Cascatense, pode estar relacionado com a incompatibilidade inicial observada, onde o desenvolvimento do porta-enxerto 'EMC' não foi proporcionalmente semelhante. As diferenças verificadas no crescimento da parte aérea entre as cultivares, provavelmente, foram devidos ao hábito de crescimento de cada cultivar, porém não se descarta a hipótese de estar relacionada com o maior estresse ocorrido em função da incompatibilidade com o porta-enxerto EMC.

\section{CONCLUSÕES}

De acordo com os resultados, conclui-se que as pereira cvs. Carrick e Seleta tem, boa compatibilidade com o marmeleiro porta-enxerto, cv. EMC, até o terceiro ano após a enxertia;

A cultivar Cascatense apresentou incompatibilidade com o marmeleiro porta-enxerto, cv. EMC.

\section{AGRADECIMENTOS}

Agradecemos a CAPES e FAPERGS pelo apoio financeiro.

\section{REFERÊNCIAS}

1. BELLINI, E. La coltivazione del pero. Verona: L'Informatore Agrario, 1993, $343 \mathrm{p}$.

2. CARRERA, M. La disaffinità d'innesto delle cultivar di pero su cotogno. Rivista di Frutticoltura, Bologna, v. LVI, n. 3, p. 41-43, 1994.

3. CAMPO-DALL'ORTO, F.A.; OJIMA, M.; BARBOSA, W. et al. Variedades de pêra para o Estado de São Paulo, Campinas: Instituto Agronômico, 1996. (Boletim Técnico, 164).

4. FACHINELLO, J.C.; MUSACHI, S., ZUCCHERELLI, S. et al. Efeito da interação porta-enxerto copa no padrão isoenzimático de plantas de pereira. Revista Brasileira de Fruticultura, Jaboticabal, v. 21, n. 3, p. 288-296, 1999.

5. FONTANAZZA, G; BALDONI, L. Propagazione e miglioramento genético. In: LALATTA, F. (Ed.). Frutticoltura generale. Roma: REDA, 1992. p. 127-280.

6. HARTMANN, H.T., KESTER, D.E., DAVIES, F.T. Theoretical aspects of grafting and budding. In: Plant propagation. 5. ed. Englewood Cliffs: Prentice Hall, 1990. p. 305-348.

7. LEMOINE, J.; MICHELESI, J.C.; ALLARD, G. Techiniche di moltiplicazione per talea erbacea e semilegnosa di alcuni portinesti del pero. Rivista di Frutticoltura e di Ortofloricoltura, Firenze, v. LIX, n. 10, p. 39- 48, 1997.

8. LORETI, F. Attuali conoscenze sui principali portinnesti degli alberi da frutto - II pero. Rivista di Frutticoltura, Bologna, v. LVI, n. 9, p. 21-26, 1994

9. MACHADO, A., CONCEIÇÃO, A.R. Programa estatístico WinStat - Sistema de Análise Estatístico para Windows - versão 2.0. Pelotas, 2002.

10. MOORE, R. Graft incompatibility beteween pear and quince: the influence of metabolites of Cidonia oblonga on suspension cultures of a Pyrus communis. American Journal of Botany, Saint Louis, v. 73, p. 1-4, 1986.

11. MUSACCHI, S. La disaffinità d'innesto del pero su cotogno: ricerche su marcatori e metaboliti secondari (fenoli, glicosidi cianogeni e proteine). Bologna, 1996. 174 f. Dissertazione (Dottorato di Ricerca in Colture Arboree) - Università degli studio di Bologna, UNIBO.

12. RYUGO, R. Fruticultura: ciencia y arte. México: AGT EDITOR, 1993. $459 \mathrm{p}$.

13. SALAYA, G.F.G. Fruticultura: el potencial productivo. 2. ed. México: ALFAOMEGA, Ediciones Universidad Católica de Chile, 1999. $342 \mathrm{p}$.

14. SIMONETTO, P.R.; GRELLMANN, E.O. Comportamento de cultivares de pereira na região serrana do Rio Grande do Sul. Porto Alegre: FEPAGRO, 1999. 28 p. (Boletim Fepagro, 9).

15. VALLI, R. Arboricoltura: generale e speciale. 6. ed. Ozzone dell'Emilia (Bo): Calderini edagricole, 2002. 658 p. 